\title{
Algebraic Extensions
}

\author{
Christoph Schwarzweller \\ Institute of Informatics \\ University of Gdańsk \\ Poland
}

\author{
Agnieszka Rowińska-Schwarzweller \\ Sopot, Poland
}

Summary. In this article we further develop field theory in Mizar [1, 2], 3] towards splitting fields. We deal with algebraic extensions [4, [5]: a field extension $E$ of a field $F$ is algebraic, if every element of $E$ is algebraic over $F$. We prove amongst others that finite extensions are algebraic and that field extensions generated by a finite set of algebraic elements are finite. From this immediately follows that field extensions generated by roots of a polynomial over $F$ are both finite and algebraic. We also define the field of algebraic elements of $E$ over $F$ and show that this field is an intermediate field of $E \mid F$.

MSC: 12F05 68V20

Keywords: algebraic extensions; finite extensions; field of algebraic numbers

MML identifier: FIELD_7, version: 8.1.11 5.65.1394

\section{PReliminaries}

Let $L_{1}, L_{2}$ be double loop structures. We say that $L_{1} \approx L_{2}$ if and only if

(Def. 1) the double loop structure of $L_{1}=$ the double loop structure of $L_{2}$.

One can verify that the predicate is reflexive and symmetric.

Now we state the propositions:

(1) Let us consider rings $R, S$. Then $R \approx S$ if and only if there exists a function $f$ from $R$ into $S$ such that $f=\operatorname{id}_{R}$ and $f$ is isomorphism.

(2) Let us consider strict rings $R, S$. Then $R \approx S$ if and only if $R=S$.

Let $F_{1}, F_{2}$ be fields. Let us note that $F_{1} \approx F_{2}$ if and only if the condition (Def. 2) is satisfied. 
(Def. 2) $\quad F_{1}$ is a subfield of $F_{2}$ and $F_{2}$ is a subfield of $F_{1}$.

Now we state the proposition:

(3) Let us consider a field $F$, an extension $E$ of $F$, and a subset $T$ of $E$. Then $\operatorname{FAdj}(F, T) \approx F$ if and only if $T$ is a subset of $F$.

Let us consider a field $F$ and extensions $E_{1}, E_{2}$ of $F$. Now we state the propositions:

(4) If $E_{1} \approx E_{2}$, then $\operatorname{VecSp}\left(E_{1}, F\right)=\operatorname{VecSp}\left(E_{2}, F\right)$.

(5) If $E_{1} \approx E_{2}$, then $\operatorname{deg}\left(E_{1}, F\right)=\operatorname{deg}\left(E_{2}, F\right)$. The theorem is a consequence of $(4)$.

Let $F$ be a field and $E$ be an extension of $F$. Note that there exists an extension of $F$ which is $E$-homomorphic and there exists an extension of $F$ which is $E$-monomorphic and there exists an extension of $F$ which is $E$-isomorphic.

Let $R$ be a ring and $a, b$ be elements of $R$. One can check that the functor $\{a, b\}$ yields a subset of $R$. Let $F$ be a field, $V$ be a vector space over $F$, and $a$ be an element of $V$. Note that the functor $\{a\}$ yields a subset of $V$. Let $a, b$ be elements of $V$. Let us observe that the functor $\{a, b\}$ yields a subset of $V$. Let us note that every basis of $V$ is linearly independent.

Now we state the proposition:

(6) Let us consider a field $F$, a vector space $V$ over $F$, and a subset $X$ of $V$. Then $X$ is linearly independent if and only if for every linear combinations $l_{1}, l_{2}$ of $X$ such that $\sum l_{1}=\sum l_{2}$ holds $l_{1}=l_{2}$.

Let $F$ be a field and $E$ be an extension of $F$. Observe that every basis of $\operatorname{VecSp}(E, F)$ is non empty and $\operatorname{deg}(E, F)$ is non zero.

Let $E$ be an $F$-finite extension of $F$. Observe that every basis of $\operatorname{VecSp}(E, F)$ is finite. Let us consider a field $F$ and an extension $E$ of $F$. Now we state the propositions:

(7) $\operatorname{deg}(E, F)=1$ if and only if the carrier of $E=$ the carrier of $F$.

(8) $\operatorname{deg}(E, F)=1$ if and only if $E \approx F$. The theorem is a consequence of (7).

(9) $\operatorname{deg}(E, F)=1$ if and only if $\left\{1_{E}\right\}$ is a basis of $\operatorname{VecSp}(E, F)$. The theorem is a consequence of $(7)$.

Let $F$ be a field and $E$ be an extension of $F$. One can check that there exists a subset of $\operatorname{VecSp}(E, F)$ which is non empty, finite, and linearly independent.

Now we state the proposition:

(10) Let us consider a field $F$, an extension $E$ of $F$, and subsets $T_{1}, T_{2}$ of $E$. Suppose $T_{1} \subseteq T_{2}$. Then $\operatorname{FAdj}\left(F, T_{1}\right)$ is a subfield of $\operatorname{FAdj}\left(F, T_{2}\right)$.

Let $F$ be a field and $p$ be a polynomial over $F$. The functor $\operatorname{Coeff}(p)$ yielding a subset of $F$ is defined by the term 
(Def. 3) $\left\{p(i)\right.$, where $i$ is an element of $\left.\mathbb{N}: p(i) \neq 0_{F}\right\}$.

Let us note that $\operatorname{Coeff}(p)$ is finite. Now we state the propositions:

(11) Let us consider a field $F$, an extension $E$ of $F$, and a polynomial $p$ over $E$. Suppose Coeff $(p) \subseteq$ the carrier of $F$. Then $p$ is a polynomial over $F$.

(12) Let us consider a field $F$, an extension $E$ of $F$, and a non zero polynomial $p$ over $E$. Suppose $\operatorname{Coeff}(p) \subseteq$ the carrier of $F$. Then $p$ is a non zero polynomial over $F$. The theorem is a consequence of (11).

(13) Let us consider a ring $R$, a ring extension $S$ of $R$, an element $p$ of the carrier of PolyRing $(R)$, and an element $q$ of the carrier of $\operatorname{PolyRing}(S)$. If $p=q$, then $\operatorname{Roots}(S, p)=\operatorname{Roots}(q)$.

Let $R$ be an integral domain and $p$ be a non zero element of the carrier of PolyRing $(R)$. Note that $\operatorname{Roots}(p)$ is finite. Let $S$ be a domain ring extension of $R$. One can check that $\operatorname{Roots}(S, p)$ is finite. Let $F$ be a field and $E$ be an extension of $F$. Let us observe that there exists an extension of $E$ which is $F$-extending. Let $E$ be an $F$-finite extension of $F$. Note that there exists an $F$-extending extension of $E$ which is $F$-finite and there exists an $F$-extending extension of $E$ which is $E$-finite. Now we state the propositions:

(14) Let us consider a field $F$, an element $p$ of the carrier of $\operatorname{PolyRing}(F)$, an extension $E$ of $F$, an $E$-extending extension $U$ of $F$, an element $a$ of $E$, and an element $b$ of $U$. If $a=b, \operatorname{then} \operatorname{ExtEval}(p, a)=\operatorname{ExtEval}(p, b)$.

(15) Let us consider a field $F$, an element $p$ of the carrier of $\operatorname{PolyRing}(F)$, an extension $E$ of $F$, and an element $q$ of the carrier of $\operatorname{PolyRing}(E)$. Suppose $q=p$. Let us consider an $E$-extending extension $U$ of $F$, and an element $a$ of $U$. Then $\operatorname{ExtEval}(q, a)=\operatorname{ExtEval}(p, a)$.

Let $R$ be a ring, $S$ be a ring extension of $R$, and $a$ be an element of $R$. The functor ${ }^{@}(a, S)$ yielding an element of $S$ is defined by the term

(Def. 4) $a$.

Let $a$ be an element of $S$. We say that $a$ is $R$-membered if and only if

(Def. 5) $a \in$ the carrier of $R$.

One can verify that there exists an element of $S$ which is $R$-membered.

Let $a$ be an element of $S$. Assume $a$ is $R$-membered. The functor ${ }^{@}(R, a)$ yielding an element of $R$ is defined by the term

(Def. 6) $a$.

Let $a$ be an $R$-membered element of $S$. Let us observe that ${ }^{@}(R, a)$ reduces to $a$. Let $F$ be a field and $E$ be an extension of $F$. One can check that there exists an element of $E$ which is non zero and $F$-algebraic.

Let $a$ be an element of $F$. One can check that ${ }^{@}(a, E)$ is $F$-algebraic. 
Let $K$ be an $E$-extending extension of $F$ and $a$ be an $F$-algebraic element of $E$. Note that ${ }^{\circledR}(a, K)$ is $F$-algebraic.

\section{More on Finite Extensions}

Now we state the propositions:

(16) Let us consider a field $F$, an extension $E$ of $F$, and an $E$-extending extension $K$ of $F$. Then every linear combination of $\operatorname{VecSp}(K, F)$ is a linear combination of $\operatorname{VecSp}(K, E)$.

(17) Let us consider a field $F$, an extension $E$ of $F$, an $E$-extending extension $K$ of $F$, a subset $B_{E}$ of $\operatorname{VecSp}(K, E)$, and a subset $B_{F}$ of $\operatorname{VecSp}(K, F)$. Suppose $B_{F} \subseteq B_{E}$. Then every linear combination of $B_{F}$ is a linear combination of $B_{E}$. The theorem is a consequence of (16).

(18) Let us consider a field $F$, an extension $E$ of $F$, an $E$-extending extension $K$ of $F$, a finite subset $B_{E}$ of $\operatorname{VecSp}(K, E)$, a finite subset $B_{F}$ of $\operatorname{VecSp}(K, F)$, a linear combination $l_{1}$ of $B_{F}$, and a linear combination $l_{2}$ of $B_{E}$. If $l_{1}=l_{2}$ and $B_{F} \subseteq B_{E}$, then $\sum l_{1}=\sum l_{2}$.

PROOF: by induction on card(the support of $l_{1}$ ).

Let $F$ be a field, $E$ be an extension of $F, K$ be an $F$-extending extension of $E, B_{E}$ be a subset of $\operatorname{VecSp}(E, F)$, and $B_{K}$ be a subset of $\operatorname{VecSp}(K, E)$. The functor $\operatorname{Base}\left(B_{E}, B_{K}\right)$ yielding a subset of $\operatorname{VecSp}((K$ qua extension of $F), F)$ is defined by the term

(Def. 7) $\left\{a \cdot b\right.$, where $a, b$ are elements of $K: a \in B_{E}$ and $\left.b \in B_{K}\right\}$.

Let $B_{E}$ be a non empty subset of $\operatorname{VecSp}(E, F)$ and $B_{K}$ be a non empty subset of $\operatorname{VecSp}(K, E)$. One can verify that $\operatorname{Base}\left(B_{E}, B_{K}\right)$ is non empty.

Now we state the propositions:

(19) Let us consider a field $F$, an extension $E$ of $F$, an $F$-extending extension $K$ of $E$, a linearly independent subset $B_{E}$ of $\operatorname{VecSp}(E, F)$, a linearly independent subset $B_{K}$ of $\operatorname{VecSp}(K, E)$, and elements $a_{1}, a_{2}, b_{1}, b_{2}$ of $K$. Suppose $a_{1}, a_{2} \in B_{E}$ and $b_{1}, b_{2} \in B_{K}$. If $a_{1} \cdot b_{1}=a_{2} \cdot b_{2}$, then $a_{1}=a_{2}$ and $b_{1}=b_{2}$.

(20) Let us consider a field $F$, an extension $E$ of $F$, an $F$-extending extension $K$ of $E$, a non empty, linearly independent subset $B_{E}$ of $\operatorname{VecSp}(E, F)$, and a non empty, linearly independent subset $B_{K}$ of $\operatorname{VecSp}(K, E)$. Then $\overline{\overline{\text { Base }\left(B_{E}, B_{K}\right)}}=\overline{\overline{B_{E} \times B_{K}}}$.

Proof: Define $\mathcal{P}$ [object, object] $\equiv$ there exist elements $a, b$ of $K$ such that $a \in B_{E}$ and $b \in B_{K}$ and $\$_{1}=a \cdot b$ and $\$_{2}=\langle a, b\rangle$. Consider $f$ being a function from $\operatorname{Base}\left(B_{E}, B_{K}\right)$ into $B_{E} \times B_{K}$ such that for every object 
$x$ such that $x \in \operatorname{Base}\left(B_{E}, B_{K}\right)$ holds $\mathcal{P}[x, f(x)] \operatorname{rng} f=B_{E} \times B_{K} \cdot f$ is one-to-one.

(21) Let us consider a field $F$, an extension $E$ of $F$, an $F$-extending extension $K$ of $E$, a non empty, finite, linearly independent subset $B_{E}$ of $\operatorname{VecSp}(E, F)$, and a non empty, finite, linearly independent subset $B_{K}$ of $\operatorname{VecSp}(K, E)$. Then $\overline{\overline{\operatorname{Base}\left(B_{E}, B_{K}\right)}}=\overline{\overline{B_{E}}} \cdot \overline{\overline{B_{K}}}$. The theorem is a consequence of $(20)$.

Let $F$ be a field, $E$ be an extension of $F, K$ be an $F$-extending extension of $E, B_{E}$ be a non empty, finite, linearly independent subset of $\operatorname{VecSp}(E, F)$, and $B_{K}$ be a non empty, finite, linearly independent subset of $\operatorname{VecSp}(K, E)$. Observe that $\operatorname{Base}\left(B_{E}, B_{K}\right)$ is finite.

Let $B_{K}$ be a non empty, linearly independent subset of $\operatorname{VecSp}(K, E), l$ be a linear combination of $\operatorname{Base}\left(B_{E}, B_{K}\right)$, and $b$ be an element of $K$. The functor down $(l, b)$ yielding a linear combination of $B_{E}$ is defined by

(Def. 8) for every element $a$ of $K$ such that $a \in B_{E}$ and $b \in B_{K}$ holds it $(a)=$ $l(a \cdot b)$ and for every element $a$ of $E$ such that $a \notin B_{E}$ or $b \notin B_{K}$ holds it $(a)=0_{F}$.

Let $B_{K}$ be a non empty, finite, linearly independent subset of $\operatorname{VecSp}(K, E)$. The functor down $l$ yielding a linear combination of $B_{K}$ is defined by

(Def. 9) for every element $b$ of $K$ such that $b \in B_{K}$ holds $i t(b)=\sum \operatorname{down}(l, b)$.

Let $E$ be an $F$-finite extension of $F, B_{E}$ be a basis of $\operatorname{VecSp}(E, F)$, and $l_{1}$ be a linear combination of $B_{K}$. The functor $\operatorname{lift}\left(l_{1}, B_{E}\right)$ yielding a linear combination of $\operatorname{Base}\left(B_{E}, B_{K}\right)$ is defined by

(Def. 10) for every element $b$ of $K$ such that $b \in B_{K}$ there exists a linear combination $l_{2}$ of $B_{E}$ such that $\sum l_{2}=l_{1}(b)$ and for every element $a$ of $K$ such that $a \in B_{E}$ and $a \cdot b \in \operatorname{Base}\left(B_{E}, B_{K}\right)$ holds $i t(a \cdot b)=l_{2}(a)$.

Now we state the propositions:

(22) Let us consider a field $F$, an $F$-finite extension $E$ of $F$, an $E$-finite, $F$ extending extension $K$ of $E$, a basis $B_{E}$ of $\operatorname{VecSp}(E, F)$, a basis $B_{K}$ of $\operatorname{VecSp}(K, E)$, and a linear combination $l$ of $\operatorname{Base}\left(B_{E}, B_{K}\right)$. Then $\operatorname{lift}(\operatorname{down} l$, $\left.B_{E}\right)=l$. The theorem is a consequence of (6).

(23) Let us consider a field $F$, an $F$-finite extension $E$ of $F$, an $E$-finite, $F$ extending extension $K$ of $E$, a basis $B_{E}$ of $\operatorname{VecSp}(E, F)$, a basis $B_{K}$ of $\operatorname{VecSp}(K, E)$, and a linear combination $l$ of $B_{K}$. Then $\operatorname{down} \operatorname{lift}\left(l, B_{E}\right)=l$.

(24) Let us consider a field $F$, an extension $E$ of $F$, an $F$-extending extension $K$ of $E$, a non empty, finite, linearly independent subset $B_{E}$ of $\operatorname{VecSp}(E, F)$, a non empty, finite, linearly independent subset $B_{K}$ of $\operatorname{VecSp}(K, E)$, and linear combinations $l, l_{1}, l_{2}$ of $\operatorname{Base}\left(B_{E}, B_{K}\right)$. Suppo- 
se $l=l_{1}+l_{2}$. Let us consider an element $b$ of $K$. Then $\operatorname{down}(l, b)=$ $\operatorname{down}\left(l_{1}, b\right)+\operatorname{down}\left(l_{2}, b\right)$.

(25) Let us consider a field $F$, an extension $E$ of $F$, an $F$-extending extension $K$ of $E$, a non empty, finite, linearly independent subset $B_{E}$ of $\operatorname{VecSp}(E, F)$, a non empty, finite, linearly independent subset $B_{K}$ of $\operatorname{VecSp}(K, E)$, and linear combinations $l, l_{1}, l_{2}$ of $\operatorname{Base}\left(B_{E}, B_{K}\right)$. If $l=$ $l_{1}+l_{2}$, then down $l=\operatorname{down} l_{1}+\operatorname{down} l_{2}$. The theorem is a consequence of (24).

Let us consider a field $F$, an $F$-finite extension $E$ of $F$, an $E$-finite, $F$ extending extension $K$ of $E$, a basis $B_{E}$ of $\operatorname{VecSp}(E, F)$, a basis $B_{K}$ of $\operatorname{VecSp}(K$, $E)$, and a linear combination $l$ of $\operatorname{Base}\left(B_{E}, B_{K}\right)$. Now we state the propositions:

(26) $\sum l=\sum$ down $l$.

Proof: by induction on card(the support of $l$ ).

(27) If $\sum l=0_{\operatorname{VecSp}((K \text { qua extension of } F), F)}$, then the support of $l=\emptyset$. The theorem is a consequence of (26).

Let us consider a field $F$, an $F$-finite extension $E$ of $F$, an $E$-finite, $F$ extending extension $K$ of $E$, a basis $B_{E}$ of $\operatorname{VecSp}(E, F)$, and a basis $B_{K}$ of $\operatorname{VecSp}(K, E)$. Now we state the propositions:

(28) $\operatorname{Lin}\left(\operatorname{Base}\left(B_{E}, B_{K}\right)\right)=$ the vector space structure of $\operatorname{VecSp}((K$ qua extension of $F), F)$. The theorem is a consequence of (23) and (26).

(29) $\operatorname{Base}\left(B_{E}, B_{K}\right)$ is a basis of $\operatorname{VecSp}((K$ qua extension of $F), F)$. The theorem is a consequence of $(27)$ and $(28)$.

(30) Let us consider a field $F$, an $F$-finite extension $E$ of $F$, and an $E$-finite, $F$ extending extension $K$ of $E$. Then $\operatorname{deg}(K, F)=(\operatorname{deg}(K, E)) \cdot(\operatorname{deg}(E, F))$. The theorem is a consequence of (29) and (21).

(31) Let us consider a field $F$, an extension $E$ of $F$, and an $E$-extending extension $K$ of $F$. Suppose $K$ is $F$-finite. Then

(i) $E$ is $F$-finite, and

(ii) $\operatorname{deg}(E, F) \leqslant \operatorname{deg}(K, F)$, and

(iii) $K$ is $E$-finite, and

(iv) $\operatorname{deg}(K, E) \leqslant \operatorname{deg}(K, F)$.

Proof: Set $B_{F}=$ the basis of $\operatorname{VecSp}(K, F)$. Reconsider $B_{E}=B_{F}$ as a finite subset of $\operatorname{VecSp}(K, E)$. $\operatorname{Lin}\left(B_{E}\right)=\operatorname{VecSp}(K, E)$. Consider $I$ being a subset of $\operatorname{VecSp}(K, E)$ such that $I \subseteq B_{E}$ and $I$ is linearly independent and $\operatorname{Lin}(I)=\operatorname{VecSp}(K, E)$.

Let $F$ be a field and $E$ be an $F$-finite extension of $F$. One can check that every $E$-finite, $F$-extending extension of $E$ is $F$-finite. 


\section{Algebraic Extensions}

Let $F$ be a field and $E$ be an extension of $F$. We say that $E$ is $F$-algebraic if and only if

(Def. 11) every element of $E$ is F-algebraic.

One can verify that every extension of $F$ which is $F$-finite is also $F$-algebraic.

Let $E$ be an $F$-algebraic extension of $F$. Note that every element of $E$ is $F$-algebraic. Now we state the propositions:

(32) Let us consider a field $F$, and an extension $E$ of $F$. Then $E$ is $F$-algebraic if and only if for every element $a$ of $E, \operatorname{FAdj}(F,\{a\})$ is $F$-finite.

(33) Let us consider a field $F$, an extension $E$ of $F$, and an element $a$ of $E$. Then $a$ is $F$-algebraic if and only if there exists an $F$-finite extension $B$ of $F$ such that $E$ is $B$-extending and $a \in B$.

Let $F$ be a field, $E$ be an extension of $F$, and $T$ be a subset of $E$. We say that $T$ is $F$-algebraic if and only if

(Def. 12) for every element $a$ of $E$ such that $a \in T$ holds $a$ is F-algebraic.

One can verify that there exists a subset of $E$ which is finite and $F$-algebraic. Now we state the propositions:

(34) Let us consider a field $F$, an extension $E$ of $F$, an element $b$ of $E$, a subset $T$ of $E$, an extension $E_{1}$ of $\operatorname{FAdj}(F, T)$, and an element $b_{1}$ of $E_{1}$. Suppose $E_{1}=E$ and $b_{1}=b$. Then $\operatorname{FAdj}(F,\{b\} \cup T)=\operatorname{FAdj}\left(\operatorname{FAdj}(F, T),\left\{b_{1}\right\}\right)$.

Proof: $\{b\} \cup T \subseteq$ the carrier of $\operatorname{FAdj}\left(\operatorname{FAdj}(F, T),\left\{b_{1}\right\}\right)$ by $[6,(35),(36)]$. $\operatorname{FAdj}(F, T)$ is a subfield of $\operatorname{FAdj}(F,\{b\} \cup T)$.

(35) Let us consider a field $F$, an extension $E$ of $F$, an element $b$ of $E$, a subset $T$ of $E$, an extension $E_{1}$ of $\operatorname{FAdj}(F,\{b\})$, and a subset $T_{1}$ of $E_{1}$. Suppose $E_{1}=E$ and $T_{1}=T . T$ Then $\operatorname{FAdj}(F,\{b\} \cup T)=\operatorname{FAdj}\left(\operatorname{FAdj}(F,\{b\}), T_{1}\right)$. Proof: $\{b\} \cup T \subseteq$ the carrier of $\operatorname{FAdj}\left(\operatorname{FAdj}(F,\{b\}), T_{1}\right)$ by $[6,(35),(36)]$. $\operatorname{FAdj}(F,\{b\})$ is a $\operatorname{subfield}$ of $\operatorname{FAdj}(F,\{b\} \cup T)$.

Let $F$ be a field, $E$ be an extension of $F$, and $T$ be a finite, $F$-algebraic subset of $E$. One can verify that $\operatorname{FAdj}(F, T)$ is $F$-finite.

Now we state the propositions:

(36) Let us consider a field $F$, an extension $E$ of $F$, and an $F$-algebraic element $a$ of $E$. Then $E \approx \operatorname{FAdj}(F,\{a\})$ if and only if $\operatorname{deg} \operatorname{MinPoly}(a, F)=$ $\operatorname{deg}(E, F)$. The theorem is a consequence of (5), (31), (30), and (8).

(37) Let us consider a field $F$, and an extension $E$ of $F$. Then $E$ is $F$-finite if and only if there exists a finite, $F$-algebraic subset $T$ of $E$ such that $E \approx \operatorname{FAdj}(F, T)$.

Proof: by induction on $\operatorname{deg}(E, F)$. 
Let $F$ be a field, $E$ be an extension of $F$, and $p$ be a non zero element of the carrier of $\operatorname{PolyRing}(F)$. Note that $\operatorname{Roots}(E, p)$ is $F$-algebraic.

Now we state the proposition:

(38) Let us consider a field $F$, an extension $E$ of $F$, and a non zero element $p$ of the carrier of $\operatorname{PolyRing}(F)$. Then $\operatorname{FAdj}(F, \operatorname{Roots}(E, p))$ is $F$-algebraic.

Let us consider a field $F$, an extension $E$ of $F$, and an $E$-extending extension $K$ of $F$. Now we state the propositions:

(39) If $K$ is $E$-algebraic and $E$ is $F$-algebraic, then $K$ is $F$-algebraic. The theorem is a consequence of (12), (15), and (33).

(40) If $K$ is $F$-algebraic, then $K$ is E-algebraic and $E$ is $F$-algebraic. The theorem is a consequence of (15).

\section{The Field of Algebraic Elements}

Let $F$ be a field, $E$ be an extension of $F$, and $a, b$ be $F$-algebraic elements of $E$. Observe that $\operatorname{FAdj}(F,\{a, b\})$ is $F$-finite and $0_{E}$ is $F$-algebraic and $1_{E}$ is $F$-algebraic.

Let $a, b$ be $F$-algebraic elements of $E$. One can verify that $a+b$ is $F$-algebraic and $a-b$ is $F$-algebraic and $a \cdot b$ is $F$-algebraic.

Let $a$ be an $F$-algebraic element of $E$. Let us note that $-a$ is $F$-algebraic.

Let $a$ be a non zero, $F$-algebraic element of $E$. Let us observe that $a^{-1}$ is F-algebraic.

The functor $\operatorname{Alg}-\operatorname{Elem}(E)$ yielding a subset of $E$ is defined by the term

(Def. 13) the set of all $a$ where $a$ is an F-algebraic element of $E$.

The functor Field-Alg-Elem $(E)$ yielding a strict double loop structure is defined by

(Def. 14) the carrier of $i t=\operatorname{Alg}$-Elem $(E)$ and the addition of $i t=$ (the addition of $E)\lceil$ (the carrier of $i t)$ and the multiplication of $i t=$ (the multiplication of $E) \uparrow$ (the carrier of $i t$ ) and the one of $i t=1_{E}$ and the zero of $i t=0_{E}$.

We introduce the notation $\mathrm{F}-\mathrm{Alg}(E)$ as a synonym of Field-Alg-Elem $(E)$.

Observe that $\mathrm{F}-\mathrm{Alg}(E)$ is non degenerated and $\mathrm{F}-\mathrm{Alg}(E)$ is Abelian, addassociative, right zeroed, and right complementable and $\mathrm{F}-\mathrm{Alg}(E)$ is commutative, associative, well unital, distributive, and almost left invertible and $\mathrm{F}-\mathrm{Alg}(E)$ is $F$-extending and $\mathrm{F}-\mathrm{Alg}(E)$ is $F$-algebraic. Now we state the propositions:

(41) Let us consider a field $F$, and an extension $E$ of $F$. Then $\mathrm{F}-\operatorname{Alg}(E)$ is an extension of $F$.

(42) Let us consider a field $F$, and an extension $E$ of $F$. Then $E$ is an extension of $\mathrm{F}-\mathrm{Alg}(E)$. 
(43) Let us consider a field $F$, an extension $E$ of $F$, and an extension $K$ of $E$. Then $\mathrm{F}-\mathrm{Alg}(K)$ is an extension of $\mathrm{F}-\mathrm{Alg}(E)$.

(44) Let us consider a field $F$, and an $F$-algebraic extension $E$ of $F$. Then $\mathrm{F}-\mathrm{Alg}(E) \approx E$.

\section{REFERENCES}

[1] Grzegorz Bancerek, Czesław Byliński, Adam Grabowski, Artur Korniłowicz, Roman Matuszewski, Adam Naumowicz, Karol Pąk, and Josef Urban. Mizar: State-of-the-art and beyond. In Manfred Kerber, Jacques Carette, Cezary Kaliszyk, Florian Rabe, and Volker Sorge, editors, Intelligent Computer Mathematics, volume 9150 of Lecture Notes in Computer Science, pages 261-279. Springer International Publishing, 2015. ISBN 978-3319-20614-1. doi:10.1007/978-3-319-20615-8_17.

[2] Grzegorz Bancerek, Czesław Byliński, Adam Grabowski, Artur Korniłowicz, Roman Matuszewski, Adam Naumowicz, and Karol Pąk. The role of the Mizar Mathematical Library for interactive proof development in Mizar. Journal of Automated Reasoning, 61(1):9-32, 2018. do1 $10.1007 / \mathrm{s} 10817-017-9440-6$

[3] Adam Grabowski, Artur Korniłowicz, and Christoph Schwarzweller. On algebraic hierarchies in mathematical repository of Mizar. In M. Ganzha, L. Maciaszek, and M. Paprzycki, editors, Proceedings of the 2016 Federated Conference on Computer Science and Information Systems (FedCSIS), volume 8 of Annals of Computer Science and Information Systems, pages 363-371, 2016. doi 10.15439/2016F520.

[4] Nathan Jacobson. Basic Algebra I. Dover Books on Mathematics, 1985.

[5] Serge Lang. Algebra. Springer, 3rd edition, 2005.

[6] Christoph Schwarzweller. Ring and field adjunctions, algebraic elements and minimal polynomials. Formalized Mathematics, 28(3):251-261, 2020. doi 10.2478/forma-2020-0022.

Accepted March 30, 2021 